\title{
28 Research Soure \\ Food Cravings And Personality Traits In Pregnant Women: Is There An Association?
}

Damar Prasmusinto ( $D$ masdamar@yahoo.com )

Faculty of Medicine Universitas Indonesia

Muhammad Ikhsan

Faculty of Medicine Universitas Indonesia

Martina Wiwie Setiawan

Faculty of Medicine Universitas Indonesia

Rina Agustina

Faculty of Medicine Universitas Indonesia

\section{Research Article}

Keywords: food cravings, Indonesia, neuroticism trait, personality traits, pregnancy

Posted Date: November 15th, 2021

DOI: https://doi.org/10.21203/rs.3.rs-1035186/v1

License: (c) (1) This work is licensed under a Creative Commons Attribution 4.0 International License. Read Full License 


\section{Abstract}

Background: Food craving is a common phenomenon during pregnancy, but explanation on how this behaviour can happen remains unclear. This behaviour may be influenced by personality traits that have been known to linked with obesity and addiction affecting pregnancy outcome. Therefore, we identified the prevalence of food craving and evaluated its relationship with personality traits in pregnant women.

Methods: We conducted a cross-sectional survey involving 500 healthy pregnant women in urban area of Jakarta, Indonesia. Food cravings were assessed using a translated and validated Food Craving Inventory of Indonesian version. Personality traits were determined by using a Big Five Personality Trait Short Questionnaire that has been translated and validated for Indonesians. Association of personality traits and food cravings were analysed by binary logistic regression.

Results: The mean age of pregnant women was $28.3 \pm 5.3$ years. Food craving prevalence in in this population was $63.8 \%$. The most common personality trait in this study was conscientiousness $(65.5 \%)$. Neuroticism trait was significantly associated with food cravings with OR 9.146 [95\% Cl: 2.76-30.4; pvalue $<0.05]$.

Conclusion: Large proportion of Indonesian pregnant women had food craving living in urban area of Jakarta. A high neuroticism trait has a strong association with food cravings during pregnancy that indicates potential influence of personality traits to this problem. If personality traits influence the food choice in pregnant women, it may subsequently influence nutritional intake of the pregnant women and it may affect pregnancy outcome.

\section{Background}

Pregnancy is a process that causes many physiological changes of the women. Dietary intake is one of the many changes that affect pregnancy. ${ }^{1,2}$ Evidence shows that dietary intake in humans is influenced by physiological, psychological, and cultural factors. With various things that happen during pregnancy, there may be changes in appetite especially on the emergence of feelings to eat the desired food (food cravings) ${ }^{2,3}$

Some studies suggested that food cravings have an important role in pathological health outcomes related to dietary intake and weight gain, such as overweight and obesity, binge eating, ${ }^{4}$ and unsuccessful weight loss. ${ }^{5-7}$ Furthermore, Allison et al ${ }^{8}$ also showed that food craving is one of the determinants of excessive prenatal energy intake and is associated with the occurrence of overweight condition during pregnancy in African women. This is also supported by studies in the United States, which found that pregnant women generally crave sweet foods such as cakes, chocolate, and sugary drinks, and this craving is associated with increased intake of sucrose, fat, and carbohydrates. ${ }^{9-11}$ Increased nutritional intake that contributes to overweight condition during pregnancy may result in unfavourable outcomes such as increasing incidence of caesarean section, gestational diabetes mellitus, 
hypertension, preeclampsia, labour complications, neonatal hypoglycaemia, perinatal complications, and impaired initiation of breastfeeding. ${ }^{12}$

Additionally, Sutin et $\mathrm{al}^{13}$ found that a person's personality trait may affect dietary intake and weight gain. Currently, it is suggested that there are five core personality traits, namely openness, conscientiousness, extraversion, agreeableness, and neuroticism. From the previous studies, it was found that certain personality traits such as neuroticism have impulsive tendencies that are associated with obesity, ${ }^{14}$ food addiction, ${ }^{15}$ binge eating, ${ }^{16}$ alcohol consumption, ${ }^{17}$ smoking, ${ }^{18}$ and substance abuse. ${ }^{19}$ On the contrary, three studies showed that people with conscientiousness personality trait are less likely to be obese since they generally have a regular and healthy lifestyle such as doing regular exercise, consuming healthy foods, and avoiding alcohol intake. ${ }^{13,20,21}$ These studies generally compared personality traits with the incidence of obesity and addiction, and until now there has been no study that has linked them with food cravings. Therefore, we aim to identify the incidence of food craving and its association with personality traits in pregnant women. If personality traits influence the food choice in pregnant women, it may subsequently influence nutritional intake of the pregnant women and it may affect pregnancy outcome.

\section{Methods}

This is a cross-sectional survey study involving 500 healthy pregnant women. The women were selected based on the following inclusion criteria: (1) pregnant women any trimester who came for antenatal care (2) willing to participate in the study. The exclusion criteria of this study were (1) the age of the subjects under 21 years old; (2) pregnancy conceived from assisted reproductive technology (through ovulation induction or in vitro fertilization); (3) not able to read or communicate in Indonesian language; (3) twin pregnancy; (4) history of diabetes mellitus, chronic kidney disease, hypertension, and thyroid disease; (5) regular use of any drugs (more than 7 days) other than pregnancy supplementation; (6) active smoking status or a history of active smoking; and (7) history of alcohol consumption. Samples were taken from ten randomly selected primary health care from five districts in urban area of Jakarta, Indonesia.

The food cravings were assessed using the Food Craving Inventory ( $\mathrm{FCl}$ questionnaire. ${ }^{22} \mathrm{The} \mathrm{FCl}$ questionnaire assesses the presence or absence of food cravings and what types of food are most craved. Forward and backward translation of the questionnaire were carried out from the US version, followed by adjustments to the contents using Indonesian food. The translation result in Indonesian version was considered similar to the English version and was adjusted to the intake of 40 healthy pregnant women from outpatient clinic of Dr. Cipto Mangunkusumo National General Hospital, Jakarta, Indonesia.

The personality traits were determined based on the Big Five Personality Trait Short Questionnaire (BFPTSQ). The Indonesian version of the BFPTSQ has been validated and reliable for adult respondents (18-50 years old) with a Cronbach alpha value of 0.87 and an Aiken- $V$ index of $0.71-0.98 .{ }^{23}$ 
This study has been approved by the Ethics Commission of the Faculty of Medicine, Universitas Indonesia with the ethical clearance No: KET-286/UN2.F1/ ETIK/PPM.00.02/ 2019. Each participant was given detailed explanation about this research in advance, starting from the title, objectives, procedures, and benefits of the study. The subjects have the right to agree to participate or to refuse to participate. Each subject who was willing to participate has been asked for approval (using informed consent). Every data taken was kept confidential and the subjects have the right to know the results of this study. This research follows the ethical principles of research as stated in the Declaration of Helsinki.

The statistical analysis was carried out using SPSS software version 20. Factors such as sociodemographic, pregnancy history, body mass index, and personality traits were analysed by Chi-square test or Fisher-exact test. The prevalence of food craving was analysed descriptively and presented in the form of numbers and percentages. The association between personality traits and food cravings was analysed by chi-square and logistic regression to determine the odd ratio with $95 \%$ confidence interval.

\section{Results}

Initially, 547 pregnant women were recruited in the study. After excluding 47 women who did not meet the criteria, 500 pregnant women were included in final analysis.

Most of the subjects in this study were 21-25 years old but the mean age was $28.3 \pm 5.3$ years. Most of them were housewives and had graduated from high schools. There were at least 300 ethnic groups in Indonesia and each has its own distinctive culture. However, in this study only 9 ethnic groups were recruited, that are the most common ethnic groups in Jakarta. Javanese was the most common ethnicity in this study. Most have household income above the minimum wage in this area, which is around USD 390 / month. The mean gestational age when recruited was $20.5 \pm 9.7$ weeks, the most common of which was the first trimester. The characteristics of the subjects can be seen in Table 1.

For the type of personality traits, conscientiousness was the most frequent personality type in this study. It is followed by agreeableness, extraversion, neuroticism, and openness subsequently. Low conscientiousness personality subject was not found in this study. (Figure 1)

We observed that for every personal trait the probability of having food cravings was higher than not having one, although statistically not significant, except for neuroticism. Comparing each personality trait, high neuroticism was the personality trait that has the most frequent food cravings, followed by low extraversion and high openness. Interestingly, low neuroticism has the least food cravings. Subjects with high extraversion also have the least for having food cravings. Therefore, high neuroticism and low neuroticism traits had a significantly difference statistically $(P<0.05)$ (Table 2$)$

Subjects with neuroticism personality trait had the odds ratio (OR) of 9.146 [95 \% confidence interval (Cl): 2.76-30.35; $p$-value $<0.05$ ] to have food cravings and it was statistically significant. No statistically significant values were found for other personality traits. High extraversion personality trait seemed to have less risk of having food cravings, although the association was not significant. (Table 3) 


\section{Discussion}

In this study large number of pregnant women in urban area of Jakarta had food cravings (63.8\%). A food craving is a strong desire to consume certain foods that is very difficult to resist and specifically different from mere hunger. If the craved food is not available, a feeling of anxious will be aroused and after it is available, there is a change in speed of consumption. ${ }^{22,24}$

The phenomenon of fruit craving is common, especially to pregnant women. It is generally known that they sometimes crave sweet food, fruit, or calorie-dense foods, as well as strange combinations such as pickles and ice cream. Even pica may also occur. Although this phenomenon is widely known, it is still not properly understood. ${ }^{5}$

The concept of personality traits refers to traits consistent over time and traits influencing behavior. ${ }^{25}$ Personality is one of the psychological variables that can affect psychopathological development. ${ }^{26}$ Personality traits have been corelated with several issues such as healthy behaviour, happiness, academic and job performance, and antisocial and criminal conduct. There are several models to describe human differences in personality traits based on different perspectives. One of the most widely accepted structural personality models is the Big Five Factor which categorizes individuals in five dimensions. ${ }^{27}$

The five-dimension personality traits are openness to experience, conscientiousness, extraversion, agreeableness, and neuroticism. Each personality trait has its characteristic. People with openness to experience trait are usually curious, imaginative, and like to have new experience. Those who have conscientiousness trait have good planning, high levels of thoughtfulness, good impulse control, and goal-directed behaviour. Extraversion trait is reflected by sociability, talkativeness, activity, excitability, and positive emotionality. People with agreeableness trait have empathy, altruism, kindness, and affection behaviours. Neuroticism trait is represented with negative emotions, such as anxiety, fear, sadness, and low self-esteem. ${ }^{28}$ Every traits has its pole, for example people with high neuroticism have a lot of stress experience, while those with low neuroticism on the contrary, are emotionally stable. Whereas people with high extraversion trait like to be the centre of attention, it is difficult for those with low extraversion trait to have social interaction.

Gaining body weight and having appetite may be determined by various factors such as health status, age, gender, genetics and socioeconomic status. These will form a dietary pattern, and it can represent the regulation of emotions, as well as the inability to resist food cravings or binge eating. Evidences suggests that the manifestations of weight gain are also consistent with individual personality traits. ${ }^{13}$

Many factors influence the personality traits in populations. Gender, culture, geography, social status, and many others can form people personality traits. We observed that the number of subjects with high conscientious trait was the highest in our samples. It was different with other study in this country, which 
collected samples from college students. ${ }^{29}$ In addition, the difference was also observed in a study in Great Britain. ${ }^{30}$ High conscientious trait is associated with orderliness behaviour.

It is suggested that food cravings do not influence the total dietary intake during pregnancy, nor are they associated with more gestational weight gain, risk of maternal glycaemia or offspring outcome biometri. ${ }^{31}$ However, others found that the first trimester craving is associated with dietary intake and the type of cravings may modulate the risk of gestational diabetes melitus. ${ }^{32}$ The personality traits affect dietary intake and weight gain because these individual characteristics are related to patterns of thinking, feeling, and behaviour.

Many studies have reported on food cravings but only this study has linked cravings with personality traits in pregnancy. From all five types of personality trait, only neuroticism had a significant statistic difference in food cravings. Therefor women with high neuroticism trait should receive special attention when getting pregnant. High neuroticism trait is represented by irritable behaviour which is having a lot of stress, easy to be angry, anxious, and always trying to exact revenge. Individuals with high neuroticism trait are easily threatened, have difficulties to deal with problems, and often experience negative emotions such as fear, shame, and anger. In correlation with the incidence of mental and physical health problems, a meta-analysis study conducted by Malouff et al ${ }^{33}$ found a strong correlation of high neuroticism with it, in comparison with other personality traits. Therefore, it seems that this personality trait more easily gets food cravings since food cravings usually reflect an unusual habit that cannot be controlled. On the contrary, low neuroticism personal trait has a characteristic of stable emotion, having ability to manage stress, happiness, and optimistic. From a previous study, it was found that high neuroticism trait had impulsive tendency to food addiction. ${ }^{15}$ In addition, this personality trait was also associated with obesity, binge eating, alcohol consumption, smoking, and substance abuse. ${ }^{14,16-19}$

Individuals with the neuroticism personality trait are said to be easier to give in to temptation to eat and have difficulty in following the discipline of healthy living habits such as doing exercise or physical activity. In addition, they also have difficulty in following a strict schedule at meal times, so it will be difficult to reduce extra calorie intake outside of mealtimes. ${ }^{14}$ Another study also found that high neuroticism trait has higher triglyceride level and higher inflammation. ${ }^{34,35}$

In previous studies, the personality opposite to neuroticism is the conscientiousness personality trait, and people with this trait are rarely obese. This personality trait is known to have a regular, disciplined, and healthy lifestyle such as doing regular exercise, eating healthy foods, and avoiding alcohol. This is also reflected in other studies which show that people with conscientiousness personalities have lower levels of fat. They will have more organized and scheduled time to eat and healthy choice of food. They also able to resist cravings such as drinking alcohol and binge eating. ${ }^{13,14,20,21}$

The weakness of this study is the subjective questionnaire relies on the patient's memory so that recall bias may be occurred. Personality traits assessment instrument is based on self-assessment that is done in a short time and not giving enough time to observe the subject. However, the implication of this study 
that if we detect personality traits of women before pregnant, we should give an appropriate antenatal care for the food cravings' women, especially with high neuroticism trait, to prevent adverse pregnancy outcome as reported above. Further research is suggested to evaluate the association of personality traits, food craving, and pregnancy outcome.

\section{Conclusion}

Large proportion of Indonesian pregnant women had food craving living in urban area of Jakarta. A high neuroticism trait has a strong association with food cravings during pregnancy that indicates potential influence of personality traits to this problem. If personality traits influence the food choice in pregnant women, it may subsequently influence nutritional intake of the pregnant women and it may affect pregnancy outcome.

\section{Declarations}

\section{Ethics approval and consent to participate}

This study has been approved by the Ethics Commission of the Faculty of Medicine, Universitas Indonesia with the ethical clearance No: KET-286/UN2.F1/ ETIK/PPM.00.02/ 2019. Each participant was given detailed explanation about this research in advance, starting from the title, objectives, procedures, and benefits of the study. The subjects have the right to agree to participate or to refuse to participate. Each subject who was willing to participate has been asked for approval (using informed consent). Every data taken was kept confidential and the subjects have the right to know the results of this study. This research follows the ethical principles of research as stated in the Declaration of Helsinki.

\section{Consent for publication}

Not applicable

\section{Availability of data and materials}

The datasets used and/or analysed during the current study are available from the corresponding author on reasonable request.

\section{Competing interests}

The authors declare that they have no competing interests

\section{Funding}

All source of funding for the research are from authors' personal budget, including data collection, analysis, and interpretation data.

\section{Authors Contributions}


DP was the main author which provide the idea and concept of research, interpreting data, writing and editing the manuscript. MI was collecting and collaborating the data including statistical analysis and writing the manuscript. MWS provided the concept from psychiatric view, including validity the questionnaire uses for personality trait. RA provided the food craving inventory questionnaire, including validating the questionnaire, and also provided the manuscript editing. All authors have read and approved the final manuscript.

\section{Acknowledgements}

Not applicable

\section{References}

1. Brown EA, Ruvolo M, Sabeti PC. Many ways to die, one way to arrive: how selection acts through pregnancy. Trends in Genetics 2013; <background-color:\#FFCC66;bverticalalign:super;>29</background-color:\#FFCC66;bvertical-align:super;>: 585-592.

2. McKerracher $L$, Collard $M$, Henrich J. Food aversions and cravings during pregnancy on Yasawa Island, Fiji. Human Nature 2016; <background-color:\#FFCC66;bvertical-align:super;>27</backgroundcolor:\#FFCC66;bvertical-align:super;>: 296-315.

3. Patil CL, Young SL. Biocultural considerations of food cravings and aversions: an introduction. Ecology of food and nutrition 2012; <background-color:\#FFCC66; bverticalalign:super;>51</background-color:\#FFCC66;bvertical-align:super;>: 365-373.

4. Joyner MA, Gearhardt AN, White MA. Food craving as a mediator between addictive-like eating and problematic eating outcomes. Eating behaviors 2015; <background-color:\#FFCC66;bverticalalign:super;>19</background-color:\#FFCC66;bvertical-align:super;>: 98-101.

5. Orloff NC, Hormes JM. Pickles and ice cream! Food cravings in pregnancy: hypotheses, preliminary evidence, and directions for future research. Frontiers in psychology 2014; <backgroundcolor:\#FFCC66;bvertical-align:super;>5</background-color:\#FFCC66;bvertical-align:super;>: 1076.

6. Forman EM, Hoffman KL, McGrath KB, Herbert JD, Brandsma LL, Lowe MR. A comparison of acceptance-and control-based strategies for coping with food cravings: An analog study. Behaviour research and therapy 2007; <background-color:\#FFCC66;bvertical-align:super;>45</backgroundcolor:\#FFCC66;bvertical-align:super;>: 2372-2386.

7. Vander Wal JS, Johnston KA, Dhurandhar NV. Psychometric properties of the State and Trait Food Cravings Questionnaires among overweight and obese persons. Eating Behaviors 2007; <background-color:\#FFCC66;bvertical-align:super;>8</background-color:\#FFCC66;bverticalalign:super; : : 211-223.

8. Allison KC, Wrotniak BH, Paré E, Sarwer DB. Psychosocial characteristics and gestational weight change among overweight, African American pregnant women. Obstetrics and gynecology international 2012; <bvertical-align:super;>2012</bvertical-align:super;>. 
9. Pope JF, Skinner JD, Carruth BR. Cravings and aversions of pregnant adolescents. Journal of the American Dietetic Association 1992; <background-color:\#FFCC66; bverticalalign:super;>92</background-color:\#FFCC66;bvertical-align:super;>: 1479.

10. Tierson FD, Olsen CL, Hook EB. Influence of cravings and aversions on diet in pregnancy. Ecology of Food and Nutrition 1985; <background-color:\#FFCC66;bvertical-align:super;>17</backgroundcolor:\#FFCC66;bvertical-align:super;>: 117-129.

11. Belzer LM, Smulian JC, Lu S-E, Tepper BJ. Food cravings and intake of sweet foods in healthy pregnancy and mild gestational diabetes mellitus. A prospective study. Appetite 2010; <backgroundcolor:\#FFCC66;bvertical-align:super;>55</background-color:\#FFCC66;bvertical-align:super;>: 609615.

12. Council NR. Weight gain during pregnancy: reexamining the guidelines. National Academies Press, 2010.

13. Sutin AR, Rogers DL, Mercado A, Weimer A, Rodriguez CC, Gonzalez M et al. The association between personality traits and body mass index varies with nativity among individuals of Mexican origin. Appetite 2015; <background-color:\#FFCC66;bvertical-align:super;>90</backgroundcolor:\#FFCC66;bvertical-align:super;>: 74-79.

14. Sutin AR, Ferrucci L, Zonderman AB, Terracciano A. Personality and obesity across the adult life span. Journal of personality and social psychology 2011; <background-color:\#FFCC66;bverticalalign:super;>101</background-color:\#FFCC66;bvertical-align:super;>: 579.

15. Murphy CM, Stojek MK, MacKillop J. Interrelationships among impulsive personality traits, food addiction, and body mass index. Appetite 2014; <background-color:\#FFCC66; bverticalalign:super;>73</background-color:\#FFCC66;bvertical-align:super;>: 45-50.

16. Schag K, Schönleber J, Teufel M, Zipfel S, Giel KE. Food-related impulsivity in obesity and Binge Eating Disorder-a systematic review. Obesity reviews 2013; <background-color:\#FFCC66;bverticalalign:super;>14</background-color:\#FFCC66;bvertical-align:super;>: 477-495.

17. Stojek MM, Fischer S, Murphy CM, MacKillop J. The role of impulsivity traits and delayed reward discounting in dysregulated eating and drinking among heavy drinkers. Appetite 2014 ; <backgroundcolor:\#FFCC66;bvertical-align:super;>80</background-color:\#FFCC66;bvertical-align:super;>: 81-88.

18. Friedel JE, DeHart WB, Madden GJ, Odum AL. Impulsivity and cigarette smoking: Discounting of monetary and consumable outcomes in current and non-smokers. Psychopharmacology 2014; <background-color:\#FFCC66;bvertical-align:super;>231</background-color:\#FFCC66;bverticalalign:super;>: 4517-4526.

19. Sutin AR, Evans MK, Zonderman AB. Personality traits and illicit substances: The moderating role of poverty. Drug and Alcohol Dependence 2013; <background-color:\#FFCC66;bverticalalign:super;>131</background-color:\#FFCC66;bvertical-align:super;>: 247-251.

20. Jokela M, Hintsanen M, Hakulinen C, Batty GD, Nabi H, Singh-Manoux A et al. Association of personality with the development and persistence of obesity: a meta-analysis based on individual- 
participant data. obesity reviews 2013; <background-color:\#FFCC66;bverticalalign:super;>14</background-color:\#FFCC66;bvertical-align:super;>: 315-323.

21. Rush CC, Becker SJ, Curry JF. Personality factors and styles among college students who binge eat and drink. Psychology of Addictive Behaviors 2009; <background-color:\#FFCC66;bverticalalign:super;>23</background-color:\#FFCC66;bvertical-align:super;>: 140.

22. White MA, Whisenhunt BL, Williamson DA, Greenway FL, Netemeyer RG. Development and validation of the food-craving inventory. Obesity Research 2002; <background-color:\#FFCC66;bverticalalign:super;>10</background-color:\#FFCC66;bvertical-align:super;>: 107-114.

23. Akhtar H, Azwar S. Indonesian adaptation and psychometric properties evaluation of the big five personality inventory: IPIP-BFM-50. Jurnal Psikologi 2019; <background-color:\#FFCC66;bverticalalign:super;>46</background-color:\#FFCC66;bvertical-align:super;>: 32-44.

24. Gendall KA, Joyce PR, Sullivan PF. Impact of definition on prevalence of food cravings in a random sample of young women. Appetite 1997; <background-color:\#FFCC66; bverticalalign:super;>28</background-color:\#FFCC66;bvertical-align:super;>: 63-72.

25. Matthews G, Deary IJ, Whiteman MC. Personality traits. Cambridge University Press, 2003.

26. Kotov R, Gamez W, Schmidt F, Watson D. Linking "big" personality traits to anxiety, depressive, and substance use disorders: a meta-analysis. Psychological bulletin 2010; <backgroundcolor:\#FFCC66;bvertical-align:super;>136</background-color:\#FFCC66;bvertical-align:super;>: 768.

27. McCrae RR, Costa PT. Personality in adulthood: A five-factor theory perspective. Guilford Press, 2003.

28. Power RA, Pluess M. Heritability estimates of the Big Five personality traits based on common genetic variants. Translational psychiatry 2015; <background-color:\#FFCC66;bverticalalign:super;>5</background-color:\#FFCC66;bvertical-align:super;>: e604-e604.

29. Schmitt DP, Allik J, McCrae RR, Benet-Martínez V. The geographic distribution of Big Five personality traits: Patterns and profiles of human self-description across 56 nations. Journal of cross-cultural psychology 2007; <background-color:\#FFCC66;bvertical-align:super;>38</backgroundcolor:\#FFCC66;bvertical-align:super;>: 173-212.

30. Rentfrow PJ, Jokela M, Lamb ME. Regional personality differences in Great Britain. PloS one 2015; <background-color:\#FFCC66;bvertical-align:super;>10</background-color:\#FFCC66;bverticalalign:super;>: e0122245.

31. Hill AJ, Cairnduff V, McCance DR. Nutritional and clinical associations of food cravings in pregnancy. Journal of Human Nutrition and Dietetics 2016; <background-color:\#FFCC66;bverticalalign:super;>29</background-color:\#FFCC66;bvertical-align:super;>: 281-289.

32. Farland LV, Rifas-Shiman SL, Gillman MW. Early pregnancy cravings, dietary intake, and development of abnormal glucose tolerance. Journal of the Academy of Nutrition and Dietetics 2015; <background-color:\#FFCC66;bvertical-align:super;>115</background-color:\#FFCC66;bverticalalign:super;>: 1958-1964.

33. Malouff JM, Thorsteinsson EB, Schutte NS. The relationship between the five-factor model of personality and symptoms of clinical disorders: A meta-analysis. Journal of psychopathology and 
behavioral assessment 2005; <background-color:\#FFCC66;bvertical-align:super;>27</backgroundcolor:\#FFCC66;bvertical-align:super;>: 101-114.

34. Sutin AR, Terracciano A, Deiana B, Uda M, Schlessinger D, Lakatta EG et al. Cholesterol, triglycerides, and the Five-Factor Model of personality. Biological psychology 2010; <backgroundcolor:\#FFCC66;bvertical-align:super;>84</background-color:\#FFCC66;bvertical-align:super;>: 186191.

35. Goodwin RD, Friedman HS. Health status and the five-factor personality traits in a nationally representative sample. Journal of health psychology 2006; <background-color:\#FFCC66;bverticalalign:super;>11</background-color:\#FFCC66;bvertical-align:super;>: 643-654.

\section{Tables}

Table 1. Characteristics of selected pregnant women in 10 primary health care of Jakarta $(n=500)$

\begin{tabular}{ll}
\hline Characteristics & $\mathrm{n}(\%)$ \\
\hline Age (years) & $341(68.2)$ \\
$21-25$ & $104(20.8)$ \\
$26-30$ & $42(8.4)$ \\
$31-35$ & $13(2.6)$ \\
$36-40$ & \\
Occupancy & $308(61.6)$ \\
Housewife & $135(27)$ \\
Employee & $31(6.2)$ \\
Teacher & $23(4.6)$ \\
Entrepreneur & $3(0.6)$ \\
Merchant & \\
Education & $299(59.8)$ \\
High School & $95(19)$ \\
Junior School & $84(16.8)$ \\
Bachelor & $21(4.2)$ \\
Primary School & $1(0.2)$ \\
Post Graduate & \\
Ethnicity & $238(47.6)$ \\
Javanese & $143(28.6)$ \\
Betawi & $81(16.2)$ \\
Sundanese & $15(3)$ \\
Malay & $23(4.6)$ \\
Others & \\
Income (according to & \\
minimum wage in the area & \\
USD 390/ month) & \\
Equal and above & $358(71.6)$ \\
Below & $142(28.4)$ \\
\end{tabular}


Gestational weeks trimester
$1^{\text {st }}$
$2^{\text {nd }}$
$176(35.2)$
$3^{\text {rd }}$
205 (41)
$119(23.8)$

\section{Prevalence}

$1^{\text {st }}$ trimester

$243(76.2)$

$2^{\text {nd }}$ trimester

$63(19.7)$

$3^{\text {rd }}$ trimester

$13(4.1)$

Table 2. Distribution of personality traits in pregnant women based on food craving

\begin{tabular}{|c|c|c|c|c|}
\hline \multirow[t]{2}{*}{ Personality traits } & & \multicolumn{2}{|l|}{ n (\%) } & \multirow[t]{2}{*}{$P$} \\
\hline & & Food craving & No food craving & \\
\hline \multirow[t]{2}{*}{ Openness } & High & $29(76.3)$ & $9(23.7)$ & 0.599 \\
\hline & Low & 61(71.7) & $24(28.3)$ & \\
\hline \multirow[t]{2}{*}{ Conscientiousness } & High & $209(71)$ & $85(29)$ & - \\
\hline & Low & 0 & 0 & \\
\hline \multirow[t]{2}{*}{ Extraversion } & High & $59(68.6)$ & $27(31.4)$ & 0.335 \\
\hline & Low & $33(76.7)$ & $10(23.3)$ & \\
\hline \multirow[t]{2}{*}{ Agreeableness } & High & $95(70.4)$ & $40(29.6)$ & 0.570 \\
\hline & Low & $57(74)$ & $20(25.9)$ & \\
\hline \multirow[t]{2}{*}{ Neuroticism } & High & $50(94.3)$ & $3(5.7)$ & $0.0001^{*}$ \\
\hline & Low & $50(68.5)$ & $23(31.5)$ & \\
\hline Total & & $319(100)$ & $181(100)$ & \\
\hline
\end{tabular}

Chi-square test. * significant $\mathrm{P}<0.05 \backslash$

Table 3. Logistic regression analysis for personality traits and food craving 


\begin{tabular}{|lllll|}
\hline Personality traits & & P value & OR & Cl 95\% \\
\hline Openness & High & 0.444 & 1.39 & $0.6-3.23$ \\
& Low & 0.455 & 1.24 & $0.7-2.19$ \\
\hline Conscientiousness & High & 0.26 & 1.37 & $0.96-2.53$ \\
& Low & - & - & -- \\
\hline Extraversion & High & 0.865 & 0.95 & $0.54-1.69$ \\
\hline Agreeableness & Low & 0.654 & 1.21 & $0.53-2.78$ \\
\hline \multirow{2}{*}{ Neuroticism } & High & 0.252 & 1.33 & $0.81-2.19$ \\
& Low & 0.258 & 1.43 & $0.77-2.67$ \\
& High & $0.0001 *$ & 9.15 & $2.76-30.35$ \\
& Low & 0.55 & 1.19 & $0.67-2.1$ \\
\hline
\end{tabular}

* statistically significant

\section{Figures}

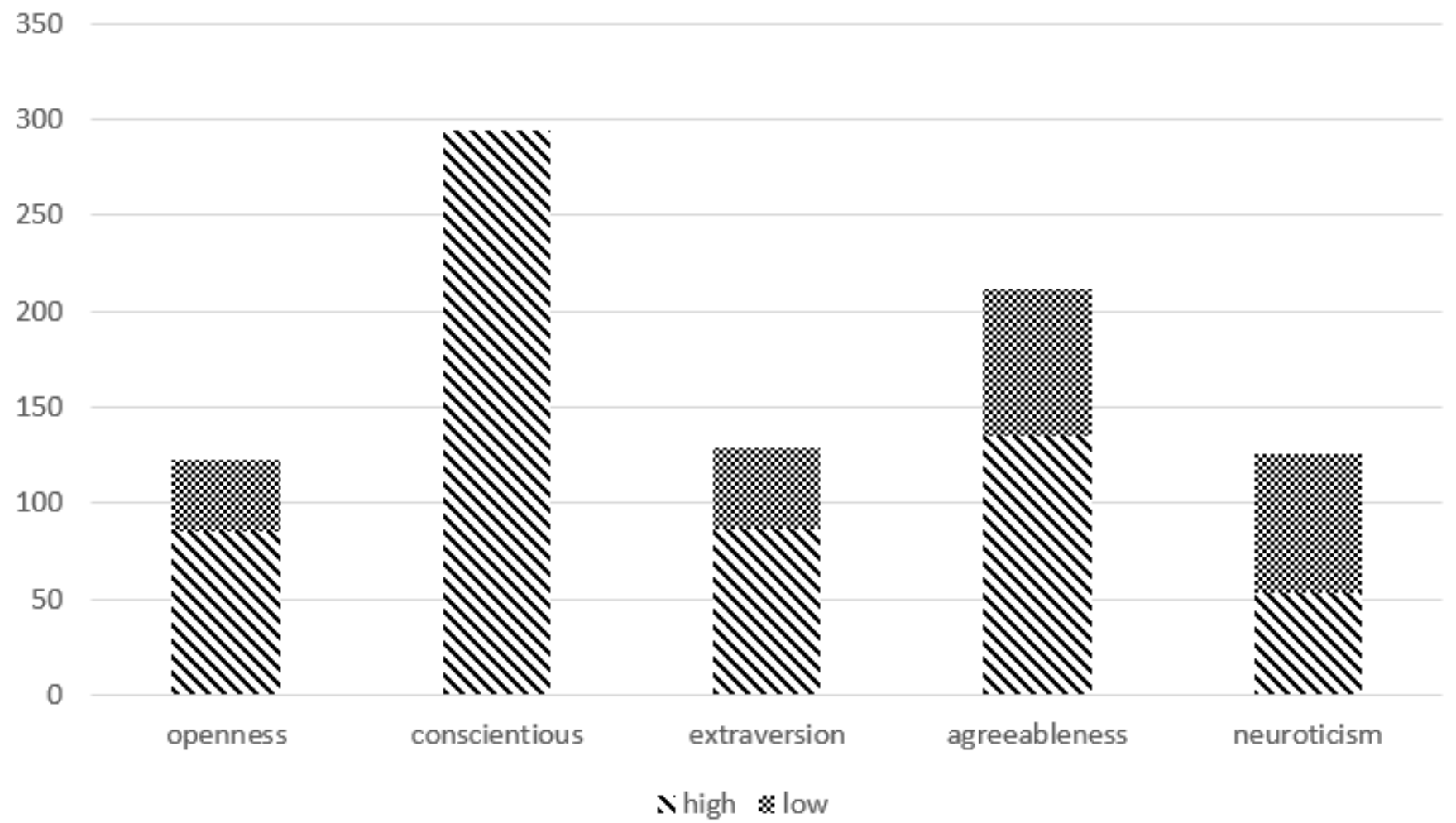

Figure 1

Distribution of personality trait in pregnant women living in Jakarta 